\title{
Editorial
}

\section{ARMS FOR CHAIRS}

\author{
Ronald J. Baker, Director
}

Institute for Departmental Leadership

Atlantic Universities

University of Prince Edward Island

While I was an assistant to the Head of English at U.B.C. in the late 'fifties and the first.Head of English at Simon Fraser in the 'sixties, many faculty "problems" disappeared. Jobs were plentiful, and the variety of jobs was increasing all the time. Faculty who thought of themselves as teachers rather than researchers could go - often at higher pay - into the new community colleges; faculty who were primarily researchers and resented heavy teaching loads in service courses could go into new graduate programs or into more prestigious institutions; faculty who for one reason or another were getting tired of teaching and research could move into the very rapidly expanding administrations, extension departments of newly created or expanded grants commissions and government departments of colleges and universities. And those who were simply tired of universities altogether could probably earn more in the very buoyant business and government world. That was in British Columbia, but the Head of one department on the Prairies told me that his problems disappeared even more rapidly than mine did in B.C. "The climate in Winnipeg is so horrible for the non-Winnipeger," he said, "that virtually anyone who is dissatisfied moves. Refuse a merit increase, refuse a promotion, assign 8:30's, insist on a section of freshmen, fail to subsidize a book, show a lack of appreciation in any way - and the "problem" is gone!" Even those who remained and were still unhappy about some things could throw their energies in to developing new courses, perhaps even new departments, and at least they always knew that they could move if they wanted.

It is unnecessary to describe all the ways in which the situation is different now. The majority of faculty cannot move at all, and often the mobile ones can move only to jobs or places they consider far less desirable. Such frustrations are, moreover, new to a high proportion of faculty; they have never known anything but expansion and opportunity. They entered graduate school with fellowships and teaching assistantships; they found it relatively easy to get better library and laboratory facilities; they were able to get faculty positions; and they were able to bargain for pay, rank, teaching loads, research facilities, and the courses they would teach and when and how they would teach them. When those histories and expectations are combined with the traditional touchiness and egocentricities of academics, the problems of a department chair are bound to increase drama- 
tically. (Stories of academic enmities and of years of fighting over a particular spot in a common room or faculty lounge or a particular office or course, etc., abound. I have heard two explanations of such faculty behaviour. First, academics mastered-easily-school, university, graduate school, etc. They traditionally deal with young people to whom they can be superior. Unlike people in business and government, they have never been frustrated in their professional preparation or lives. Consequently, the smallest frustration enrages them. Salesmen are used to not making all their sales; academics are not used to not getting firsts - or chairs, offices, etc. The second reason is that the issues involved are so trivial.

The chair's job has become more difficult for all those reasons and for others that stem from them. Relative decreases in budget and lack of mobility have led to the increased use of appeal procedures both internally and in the courts. A chair may never have seen a union contract; suddenly he may have to work with both a faculty and a non-faculty contract. Arguments and appeals based on administrative law, "fair procedures," "due process," "natural justice," human rights legislation, and labor legislation all present possible pitfalls for chairs, people who have frequently been chosen for their scholarship or teaching rather than for their administrative experience. A senior officer of the CAUT told me that he believed that a very high proportion of the disputes between individual universities and the CAUT could have been avoided, and should have been avoided, by proper administration in the department.

Many of the pitfalls I have listed above are not restricted to universi.ies. Increasing bureaucratization and legalistic administration is a feature of many areas of society today. What is peculiar to universities, I think, is the lack of help that we give people who are likely to face the problems. Until recently, universities simply did not provide any kind of in-house help to academic administrators - or to anyone else in either teaching or research for that matter. Even now, most universities do nothing or very little to help chairs with administration, and some have abandoned their efforts to help faculty with staff development in such things as reaching. Compared with government, industry, business and the services, universities have assumed that departments run themselves "naturally". They may have done that when curricula and programs remained unchanged over years and when heads were appointed essentially for life. New heads could learn from very experienced heads. Now, however, it is likely that all the chairs in a given faculty may change with only three to six year terms. If rotating heads are com. bined with rotating deans and relatively short-term presidents, it seems to some of us that we may have a kind of late Ptolemaic cosmic system - epicycles upon epicycles upon epicycles!

For the last three years, the Association of Atlantic Universities, with the help of a grant from the Kellogg Foundation, has been running series of workshops for department chairs and heads. It is administered by the unfortunately and rather pompously named Institute for Departmental Leadership based at the University of Prince Edward Island. I am the Director. The program is an adaptation of an American program by Allan Tucker. The original program can be found in his book Chairing the Academic Department. Leadership Among Peers, American 
Council of Education, Washington, 1981. (See the favorable review in the CAUT Bulletin.) The U.P.E.I. program is essentially the same, but the material has been Canadianized. Briefly, fifty chairs and heads from Atlantic universities are brought together for two four-day periods, one in early October and one in late May, at a pleasant resort on Prince Edward Island. They are grouped roughly by tens according to the size of their departments. If possible, the groups are then juggled so that there is the greatest possible mixture by institution and discipline. We do not want a group entirely of people from one university or entirely from similar disciplines. The groups typically read a chapter from the course material, answer questions on the chapter for themselves, and then discuss the material and the answers as a group. The chapter headings are:

1. Responsibilities, Roles and Powers of Department Chairpersons

2. Types of Departments, Leadership Styles, Committees, and Delegation

3. Faculty Grievances and Unions

4. Faculty Evaluation

5. Performance Counselling: Dealing with Unsatisfactory Performance

6. Assigning and Reporting Faculty Activities

7. Faculty Development: Encouraging Professional Growth

8. Departmental Decision-Making and Bringing about Change

9. Dealing with Conflict and Maintaining Faculty Morale

10. Departmental Accomplishments and Aspirations:

Setting Goals and Developing Action Plans

11. The Budget Cycle: Preparing Departmental Budget Requests and Persuading the Dean

12. Managing Departmental Resources: Time, People and Money

The material is not intended to tell people how anything should be done. It tries to describe several ways in which something might be done and then expects the group to pool its experience and wisdom to discuss the suggestions. (Under the Kellogg grant we have also brought to each session two faculty from Quebec, two from Ontario and two from Western Canada. They are expected to participate fully, but also to report back to their universities on the usefulness of the program).

By three measures the program has been successful. First, the evaluations distributed at the end of each session have been almost without exception favourable or very favourable. Second, nearly everyone who has been able to return for the second session has done so. Third, after a good deal of initial suspicion that the program was "sponsored by management and introducing American business techniques," we now find that we have plenty of volunteers, sometimes more than we can take from one institution. We are also beginning to get people from other areas of Canada. The CAUT has twice sent representatives and appears to be sympathetic to the workshops. Recently the Association of Atlantic Universities has agreed to continue the workshops after the grant runs out, even though that will mean that the cost to the individual university will go up from something like $\$ 220.00$ per person per session (for the course and full room and board) to something like $\$ 750.00$ per person per session. 
I am now quite convinced that we must do much more to help chairs and that there are at least three kinds of things that should be done. To begin with, all institutions should have short programs for new chairs, programs that focus on some of the particular administrative procedures of their own universities, some of the possible problems with contracts or legislation in the university and in the region, and above all on information about help within the institution. A number of universities have such programs. McMaster's has impressed me.

Second, chairs, particularly of service departments, may frequently need help with a particular disciplinary problem. Obviously, the various associations of chairs of departments (the Canadian Association of Chairmen of Departments of English and the Canadian Council of University Biology Chairmen, for example), must provide the machinery for such questions. How should we teach, how can we teach, composition? Should genetics be taught in a number of departments or in only one, etc.? Where there is no formal association of chairs, similar things can be done informally within the Learned Societies.

Third, there is the kind of thing that we are trying to do at our workshops. When asked to describe it very briefly, I say that it provides an opportunity for a chair to get away for a few days and think about being a chairman or chairwoman rather than thinking about being a chemist or a professor of English. It is an opportunity to think about being a chair without feeling guilty that one is not in the library or the lab or the classroom - or putting out the latest brush fire. A side benefit, of course, is we are building up a network of support among chairs. I have regularly been amazed at the fact that chairs from even small universities frequently do not know other chairs in the same university. Meeting one's colleagues from the same university is undoubtedly useful, but I do think that one of the great virtues of the present program is that it mixes people from different disciplines and different universities. If the same program were mounted for one university, I think that there is a danger that old enemies would simply be given new things to fight about. Moreover, in the problem sessions, people might be inhibited from revealing problems in their own departments or discussing particular people even if unnamed in their own departments. No one wants to admit to a group of fellow department chairs that professor $\mathrm{X}$ gave up teaching, scholarship, and research some years ago, has twenty years to go before retirement, and is talking about challenging mandatory retirement! Even though he also runs a farm!

In spite of all the criticisms, the department remains the basic unit of the university. No one would ever want to see departments run like army units, government departments or multi-nationals. Nevertheless, we must do something to help chairs now that times are very difficult and likely to remain so. The trouble with our traditional practice of throwing people in to see whether they sink or swim is that some of them sink! And having sunk, they may leave behind a costly lawsuit - and worse, a ravaged, divided department, faculty, or university. Academics are polemical, they have long memories, and some appear to have unlimited time to nurse imagined - or real - grievances. 


\title{
DU “MUSCLE” POUR LE DIRECTEUR DE DEPARTEMENT
}

\author{
Ronald J. Baker, Directeur \\ Institute for Departmental Leadership \\ Universités de l'Atlantique-Université de l'Ile du Prince-Edouard
}

Lorsque j'étais adjoint au directeur du Département d'anglais à $U B C$ à la fin des années 50, puis premier directeur du Département d'anglais à Simon Fraser à la fin des années 60 , plusieurs problèmes de corps professoral disparurent. Les emplois étaient abondants et leur variété grandissait sans cesse. Les membres du corps professoral qui se voulaient des professeurs plus que des chercheurs pouvaient aller (souvent à des salairés plus élevés) dans les nouveaux collèges communautaires. Ceux qui faisaient principalement de la recherche, et à qui déplaisaient les lourdes charges professorales en cours de services, pouvaient aller dans des programmes d'études supérieures ou dans des institutions plus prestigieuses. D'autres qui, pour une raison ou pour une autre, étaient fatigués d'enseigner ou de faire de la recherche, pouvaient déménager dans les secteurs administratifs en expansion, dans les départements d'éducation continue, dans les grands et récents organismes subventionnaires, et dans la haute administration des collèges et universités. Ceux enfin qui étaient tout simplement fatigués des universités en général pouvaient probablement gagner plus dans le remuant monde des affaires et dans le secteur gouvernemental. Ceci se passait en Colombie Britannique. Mais le directeur d'un département des Prairies me confia que ses problèmes disparaissaient encore plus rapidement que les miens. "L'ambiance à Winnipeg est si affreuse pour les non-Winnipegois qu'à peu près tout mécontent s'en va" disait-il. "Refusez une augmentation au mérite, refusez une promotion, faites commencer les cours à $8 \mathrm{~h} 30$, mettez l'accent sur une section de commençants, ne subventionnez pas un livre, montrez un manque d'appréciation quelconque, et le "problème" a disparu!" Même ceux qui restaient et étaient encore insatisfaits pouvaient dépenser leurs énergies à développer de nouveaux cours, peut-être même de nouveaux départements; et de plus, ils savaient qu'ils pouvaient toujours partir s'ils le voulaient.

Il est inutile de décrire à quel point la situation est maintenant différente. La majorité des professeurs sont bloqués, et souvent, ceux qui sont encore mobiles, peuvent seulement se tourner vers des emplois ou des endroits qu'ils considèrent bien moins attrayants. De plus, ces frustrations sont nouvelles pour une grande majorité de professeurs qui n'ont jamais connu autre chose que la croissance et la facilité. Ils ont abordé les études supérieures avec des bourses et des subventions d'auxiliaires d'enseignement, ils pouvaient assez facilement avoir de meilleurs bibliothèques et équipements de laboratoire. Ils pouvaient accéder à des postes de professeur et discuter leur salaire, leur rang, les charges de cours, les conditions de recherche, choisir leurs cours, quand et comment il les dispenseraient. Lorsque la vieille histoire et les attentes sont combinées avec le tempérament tradition. nellement susceptible et égocentrique des académiques, les problèmes d'un 
directeur de département augmentent inévitablement de façon dramatique (les histoires de rivalités académiques et d'années de luttes sur un point particulier dans les salles communautaires, salons de professeurs, bureaux personnels ou cours abondent). On m'a donné deux explications sur un tel comportement. Premièrement, les académiques ont franchi facilement les étapes de l'école, l'université et l'école supérieure. De façon générale, ils ont affaire à de jeunes gens auxquels ils sont supérieurs. Contrairement aux hommes d'affaires et aux dirigeants, ils n'ont jamais été frustrés dans leur formation ou leur vie. En conséquence. La plus petite frustration les enrage. Les vendeurs sont habitués à ne pas compléter toutes leurs ventes mais les académiques ne sont pas habitués à ne pas être les premiers (ou directeurs, officiers, etc.). La seconde raison est que les problèmes en cause sont insignifiants.

La tâche de directeur est devenue plus difficile pour toutes les raisons suivantes et leurs conséquences. Les baisses de budget et le manque de mobilité ont mené à un recours croissant aux procédures d'appel internes et devant les tribunaux. Un directeur peut n'avoir jamais vu une convention collective et devoir soudain travailler avec une législation mixte. Les plaidoyers et les appels basés sur le droit administratif, "les procédures équitables", "les principes de justice naturelle", la législation des droits de l'homme et le droit du travail, présentent tous des pièges pour les directeurs; ces derniers ayant d'ailleurs été choisis plus pour leurs qualités d'érudits ou d'enseignants que pour leur expérience en administration. Un officier supérieur de l'ACPU m'a souligné qu'il croyait qu'une grande proportion des différends entre les universités et l'ACPU pouvait être évitée, et devrait l'être par une administration adéquate du département.

Plusieurs des pièges énumérés ci-dessus ne se limitent pas aux universités. La bureaucratisation grandissante et l'administration légaliste sont des caractéristiques de plusieurs secteurs de notre société actuelle. Ce qui est particulier aux universités, je pense, est le manque de soutien aux personnes susceptibles d'affronter les problèmes. Jusqu'à récemment, les universités ne fournissaient aucune sorte d'aide interne aux administrateurs académiques (ou à n'importe qui d'autre en la matière, en enseignement ou recherche). Mème maintenant beaucoup d'universités ne font rien ou très peu pour aider les directeurs à gérer, et plusieurs ont abandonné leurs efforts pour fournir du développement professionnel à leurs professeurs. En se basant sur le gouvernement, l'industrie, les affaires et les services, les universitès ont présumé que les départements se dirigeaient eux-mêmes "naturellement". Ils pouvaient faire cela lorsque l'ensemble des programmes restait inchangé pendant des années et que les dirigeants étaient nommés quasiment à vie. Les nouveaux dirigeants pouvaient s'informer auprès d'autres dirigeants très expérimentés. Maintenant cependant, tous les directeurs, dans une faculté donnée, peuvent changer après un mandat de trois ou six ans seulement. Si la rotation des dirigeants se combine avec celle des doyens et des présidents nommés pour un assez court terme, il semble que nous nous acheminions vers un système cosmique ptolémique (épicycles sur épicycles sur épicycles!). 
Ces trois dernières années, l'Association des Universités de l'Atlantique, avec l'aide d'une subvention de la Fondation Kellog, a dirigé une série d'ateliers de travail pour les directeurs et officiers de département. Ceci est administré par l'Institute for Departmental Leadership, dont le nom peut malheureusement paraître pompeux, et qui est situé à l'Ile du Prince-Edouard. J'en suis le directeur. Le programme est une adaptation de celui de l'américan Allan Tucker. Le programme original se trouve dans son livre "Chairing the Academic Department. Leadership among Peers", American Council of Education, Washington, 1981 (Voir la critique favorable dans le Bulletin de l'ACPU). Celui de l'Université du Prince-Edouard est à peu près pareil, mais le contenu a été canadianisé. En bref, 50 directeurs et officiers d'universités de l'Atlantique se réunissent deux fois quatre jours, une fois au début d'octobre et l'autre à la fin de mai, dans un hôtel agréable de IPE. Ils sont regroupés par dizaines selon la taille de leur département. Si possible, les groupes sont organisés de façon à obtenir la plus grande variété d'institu'tions et de disciplines. Nous ne voulons pas faire de groupes constitués entièrement de personnes d'une même université ou de disciplines similaires. Typiquement, les groupes lisent un chapitre du matériel didactique, répondent individuellement aux questions sur le chapitre, et discutent ensuite en groupe sur le matériel et les questions. Les titres de chapitres sont:

1. Responsabilités, rôles et pouvoirs des directeurs de département

2. Types de départements, styles de leadership, comités et délégation

3. Griefs et syndicats de professeurs

4. Evaluation du corps professoral

5. Evaluation du rendement: que faire en cas de rendement insuffisant

6. Distribution des tâches et rapport d'activités du corps professoral

7. Incitation des professeurs au développement professionnel

8. Processus de décision départemental et introduction au changement

9. Conflit et maintien du moral du corps professoral

10. Réalisations départementales et aspirations: définir des objectifs et développer des plans d'action

11. Budget: préparer les demandes budgétaires du département et persuader le doyen

12. Gestion des ressources départementales: personnel, temps et argent

Ce cours n'a pas pour but de fournir des solutions ä toutes choses. Il tente de décrire plusieurs façons de procéder, et attend ensuite du groupe la mise en commun d'expérience et de sagesse pour discuter des suggestions (avec l'aide de la Fondation Kellog, nous avons invité à chaque session, 2 professeurs du Québec, de l'Ontario et de l'Ouest Canadien. Ils devaient participer pleinement, mais aussi faire rapport à leur université sur l'utilité du programme).

Le programme fut un succès sous trois aspects. Premièrement, les évaluations faites à la fin de chaque session étaient presque toutes favorables ou très favorables. Deuxièmement, presque tous ceux qui avaient la possibilité de suivre une autre 
session le firent. Troisièmement, après de sérieux doutes sur le fait que le programme "était parrainé par des administrateurs et voulait introduire les techniques américaines du secteur des affaires", nous trouvons maintenant beaucoup de volontaires, quelques fois plus que nous pouvons en prendre d'une institution. Nous commençons également à avoir des gens d'autres parties du Canada. L'ACPU a envoyé deux fois des représentants et semble favorable aux ateliers de travail. Récemment, l'Association des Universités de l'Atlantique a accepté de poursuivre les ateliers une fois la subvention terminée, même si cela doit se traduire pour les universités par une augmentation du coût de la session par personne (pour le cours et l'hébergement) de $220 \$$ à quelque $750 \$$.

Je suis d'ores et déjà presque convaincu que nous pouvons faire plus pour aider les directeurs et qu'il y a au moins trois avenues de solution. Pour commencer, toutes les institutions devraient avoir de courts programmes pour les nouveaux directeurs, programmes principalement axés sur quelques procédures administratives qui leurs sont particulières, quelques problèmes de contrats ou de législation universitaire ou régionale, et pardessus tout, de l'information sur l'aide disponible à l'intérieur de l'institution. Certaines universités ont de tels programmes. McMaster m’a impressionné.

Deuxièmement, les directeurs, particulièrement de départements de services, peuvent fréquemment avoir besoin d'aide pour un problème disciplinaire particulier. Evidemment, les diverses associations de directeurs de département ("The Canadian Association of Chairmen of Departments of English" et "The Canadian Council of University Biology Chairmen" par exemple) doivent fournir les mécanismes de solutions à de telles questions. Comment devrions-nous enseigner; comment pouvons-nous enseigner? La génétique devrait-elle être enseignée dans certains départements ou un seulement? etc... Là où il n'y a pas d'association formelle de directeurs, la même chose peut être faite informellement via les Sociétés savantes.

Troisièmement, il y a ce que nous essayons de faire dans nos ateliers. Lorsqu'on m'en demande une brève description, je réponds qu'ils fournissent à un directeur l'occasion de s'éloigner pour quelques jours et penser comme un directeur ou une directrice plutôt que comme un chimiste ou un professeur d'anglais. C'est également l'occasion d'agir comme directeur sans se sentir coupable de ne pas travailler à la bibliothèque, dans le laboratoire ou dans la salle de classe (ou régler les derniers problèmes brûlants). Evidemment, comme bénéfice marginal, nous sommes en train d'établir un réseau de soutien entre les directeurs. J'ai souvent été étonné par le fait que les directeurs, même de petites universités, ne connaissaient pas les autres directeurs de la même université. Rencontrer ses collèques du même établissement est évidemment utile, mais je pense que l'un des grands avantages de ce programme est de faire se rencontrer des gens de différentes disciplines et institutions. Si le même programme était mis sur pied par une université, je pense qu'il serait dangereux que de vieux ennemis se donnent tout simplement de nouvelles raisons de se battre. De plus, lors de l'étude des problèmes, les gens 
se défendraient de révéler les problèmes de leur propre département ou discuter de personnes en particulier, même de façon anonyme. Personne ne ferait admettre à un groupe de directeurs de département que le professeur $\mathrm{X}$ a cessé l'enseignement, le perfectionnement et la recherche il y a quelques années, qu'il lui reste 20 ans avant la retraite, et qu'il parle de remettre en question la retraite obligatoire! Même s'il dirige également une ferme!

En dépit de toutes les critiques, le département reste l'unité de base de l'université. Personne ne voudrait voir les départements dirigés comme des unités de l'armée, des départements du gouvernement ou des multi-nationales. Néanmoins, nous devons faire quelque chose pour aider les directeurs en ces temps difficiles qui risquent de durer encore longtemps. Le problème, avec notre technique habituelle de faire plonger les gens pour voir s'ils coulent ou surnagent, est que quelques-uns coulent! Ces noyés peuvent avoir lajssé derrière eux de coûteuses poursuites judiciaires (et pire, un département, une faculté ou une universite ravagés et divisés). Les académiques sont polémiques, ils ont la mémoire longue, et quelques-uns semblent avoir le temps d'entretenir indéfiniment d'imaginaires (ou réelles) doléances. 